\title{
Is Dopamine an Iatrogenic Disruptor of Thyroid and Cortisol Function in the Extremely Premature Infant?
}

\author{
Sze M. Ng, ${ }^{1,2}$ Gabriella Watson, ${ }^{1}$ Mark A. Turner, ${ }^{1}$ \\ Paul Newland, ${ }^{3}$ and A. Michael Weindling ${ }^{1}$ \\ ${ }^{1}$ Department of Women's and Children's Health, Institute of Translational Medicine, University of Liverpool, \\ Liverpool Women's Hospital, Crown Street, Liverpool L87SS, UK \\ ${ }^{2}$ Department of Paediatrics, Southport and Ormskirk NHS Trust, Wigan Road, Ormskirk L39 2AZ, UK \\ ${ }^{3}$ Department of Biochemistry, Alder Hey Children's Foundation Trust, Eaton Road, Liverpool L12 2AP, UK
}

Correspondence should be addressed to Sze M. Ng; may.ng@nhs.net

Received 6 April 2014; Revised 24 May 2014; Accepted 26 May 2014; Published 4 June 2014

Academic Editor: Wayne Brake

Copyright (C) 2014 Sze M. Ng et al. This is an open access article distributed under the Creative Commons Attribution License, which permits unrestricted use, distribution, and reproduction in any medium, provided the original work is properly cited.

\begin{abstract}
Background. Dopamine is frequently used as an inotropic and vasoactive agent in neonatal intensive care units. Recent studies have reported that treatment with dopamine is associated with hypothyroxinaemia of prematurity. Objectives. The aim of this study was to determine if dopamine treatment in extremely premature infants altered thyroid and cortisol function. Methods. We prospectively measured plasma cortisol, TSH, free T4, total T4, and free triiodothyronine concentrations in babies born below 28 weeks' gestation within 5 days of birth, who were either treated with dopamine (D+) or who did not receive any dopamine (D-) within 12 hours of birth. Clinical Risk Index for Babies scores, lowest mean arterial pressure and highest plasma lactate concentrations in the first 12 hours, were recorded. Results. There were 78 babies included in the study (43 males). Mean gestational age was 25 weeks and 3 days (SD 1.3 weeks). Univariate analyses showed significant differences in cortisol and thyroid function between $\mathrm{D}+$ and $\mathrm{D}-$. Multivariable analyses showed that dopamine, gestation, and CRIB were independent factors affecting FT4 concentrations. No independent factors were shown to affect cortisol or TSH concentrations. Conclusion. Dopamine administration appeared to affect FT4 concentrations but not cortisol concentrations. The mechanisms are unclear but the effect does not appear to be related to hypotension or tissue underperfusion.
\end{abstract}

\section{Introduction}

Derangement of thyroid function in extreme prematurity has been commonly described $[1,2]$. Preterm infants are also frequently hypotensive within the first 24 hours of birth. Dopamine, which is a natural catecholamine, is often used as a first line inotropic and vasoactive agent in neonatal intensive care. Dopamine has been reported in human studies to cause suppression of anterior pituitary hormone secretion $[3,4]$ and animal experiments have reported that basal secretion of TSH from pituitary thyrotrophs was significantly inhibited by dopamine in a dose-dependent manner in adult species, although no work has been done on embryonic or fetal animals $[5,6]$. Infants with severe thyroidal disruption are at an increased risk of abnormal neurodevelopment [7, 8] and dopamine has been associated with hypothyroxinaemia of prematurity $[4,9,10]$. In a randomised controlled trial of dopamine versus dobutamine, dopamine administration was associated with reduced serum concentrations of growth hormone, TSH, T4, and prolactin after 12 hours of administration in very low birth weight infants [3]. The apparent effect of dopamine as a potent inhibitor of growth hormone and prolactin was reversed on withdrawal of the dopamine [11]. The mechanisms by which this occurs in humans are not well understood and the later consequences of such iatrogenic disruption of the endocrine functions have not been studied. The aim of this study was to determine if dopamine treatment of extremely premature infants was associated with altered thyroid and cortisol concentrations in this group. 


\section{Methods}

We prospectively measured early morning blood samples which were taken as part of routine clinical sampling, which was done by phlebotomists at around 09:00. The following were measured in infants born below 28 weeks' gestation within 5 days after birth: plasma concentrations of cortisol, thyroid stimulating hormone (TSH), free thyroxine (FT4), total thyroxine (TT4), and triiodothyronine (TT3). All infants were consecutively enrolled if they were born below 28 weeks' gestation. Infants born to mothers with known thyroid or adrenal disease, on antithyroid medications or amiodarone, infants with major congenital or chromosomal abnormalities known to affect thyroid or cortisol function, were excluded. Infants were classified as exposed to dopamine $(\mathrm{D}+)$ or not exposed to dopamine (D-) within 12 hours of birth. Infants who were treated with dopamine after 12 hours of life were excluded from the study. Within infants in D+ group, dopamine infusion was present during blood sampling. Clinical Risk Index for Babies (CRIB) score [12], which is a risk-adjustment instrument widely used in neonatal intensive care, was recorded for each infant. Lowest mean arterial pressure (MAP) and highest blood lactate concentrations in the first 12 hours were also recorded. All babies were receiving intensive care support but were in a stable state. Blood pressure was monitored by a transducer attached to an indwelling arterial catheter. Systolic blood pressure (SBP), diastolic blood pressure (DBP), and mean blood pressure (MBP) were measured directly. The standard calculation for $\mathrm{MBP}$ is $[(2 \times \mathrm{DBP})+\mathrm{SBP}] / 3$. The $\mathrm{MBP}$ is generated automatically by monitors. Blood lactate was measured by arterial blood sampling taken during routine clinic care. Plasma thyroid and cortisol profiles were measured using DPC Immulite 2000 using a solid phase 2 site chemiluminescent immunometric assay.

Distributions of continuous outcomes were checked and $P$ values were calculated using Student's $t$-test for comparison of continuous parametric outcomes and Mann-WhitneyWilcoxon test for nonparametric outcomes. Comparisons of infants exposed to dopamine and infants not exposed to dopamine treatment were made using univariate analyses. Multivariable regression analysis was used to determine the independent factors that were associated with FT4 concentrations on univariate analysis. The SPSS statistical package was used for data analysis. The study was approved by Central Manchester Research Ethics Committee of the United Kingdom. The parents of eligible babies were informed of the study's objectives and informed parental consent was obtained.

\section{Results}

There were 78 babies recruited into the study (43 males). Mean $\left( \pm\right.$ SD) gestation was $25( \pm 1.3)$ weeks (range $22^{+0}$ $\left.27^{+6}\right)$. Mean $( \pm S D)$ birth weight was $815( \pm 168)$ grams. Table 1 shows the characteristics of infants on dopamine treatment compared to those who did not receive inotropes. There were 35 infants who received a dopamine infusion within 12 hours of birth. The reason for the treatment with dopamine was hypotension within the first 12 hours of life and the mean $( \pm \mathrm{SD})$ dopamine infusion administered was $8.89( \pm 3.08) \mathrm{mcg} / \mathrm{kg} / \mathrm{min}$ (range $3-15 \mathrm{mcg} / \mathrm{kg} / \mathrm{min})$. None of the infants received hydrocortisone for hypotension. There was no significant difference between methods of delivery, that is, caesarian or vaginal delivery in the two groups. Univariate analyses showed significant differences in FT4, TT4, and TT3 plasma concentrations according to dopamine exposure status, but no difference was noted in cortisol concentrations. Multiple regression analyses showed that plasma FT4 concentrations were independently associated with dopamine, gestation, and CRIB score, but not plasma lactate concentration or MAP (Table 2). Multiple regression analyses of factors affecting plasma cortisol and TSH concentrations were also performed using similar variables. None of these variables were associated with plasma cortisol or TSH concentrations. The mean timing of blood samples was day 3.8 and this had no impact on the regression analyses.

\section{Discussion}

In this study, extremely premature infants born below 28 weeks' gestation who received dopamine had significantly lower plasma FT4 levels compared with those who did not receive any treatment for hypotension. The babies given dopamine also had higher CRIB scores and lower gestation and therefore were also significantly more unwell; thus reduced plasma FT4 concentrations were also related to the severity of illness. This was also consistent with the increased plasma cortisol levels noted in the infants receiving dopamine suggesting that the hypothalamic-pituitary axis was intact and able to mount an adequate response during illness even in these very immature infants. The present findings confirm the observations of the study by Williams et al. that low plasma concentrations of thyroid hormones in infants born significantly prematurely were associated with severity of postnatal illnesses and usage of other medications. In addition to the effects of gestational and postnatal age, associations have been reported between decreased thyroid function and disease severity, infection, dexamethasone, and diamorphine administration [13]. In order to separate out the independent risks of illness or immaturity from a drugspecific effect even with the use of statistical adjustments, a multiple regression analysis suggested that dopamine administration was an independent factor associated with low FT4 levels in these very preterm infants. In the clinical setting, this is important as thyroid homeostasis also controls important functions like lung surfactant secretion, gut motility, cardiac function, and bone maturation. Furthermore, in term infants, thyroxine deficiency is associated with an increased risk of neurological damage [14].

Dopamine receptors are expressed in the thyroid, pituitary, and hypothalamus, and dopamine has been reported in clinical studies to cause anterior pituitary hormone suppression $[3,4]$. Furthermore, animal experiments have shown that basal secretion of TSH from pituitary thyrotropes was significantly inhibited by dopamine in a dose-dependent manner in adults although no work has been done in embryonic or fetal animals $[5,6]$. However, in the present study, TSH 
TABLE 1: Comparison of infants exposed to dopamine and infants not exposed to dopamine treatment.

\begin{tabular}{|c|c|c|c|c|}
\hline & $\begin{array}{c}\text { Dopamine exposure } \\
(\mathrm{D}+) \\
N=35 \\
\end{array}$ & $\begin{array}{l}\text { No dopamine (D-) } \\
\qquad N=43\end{array}$ & $\begin{array}{l}\text { Difference }(95 \% \text { CI D+ } \\
\text { minus D-) }\end{array}$ & $P$ value \\
\hline \multirow{2}{*}{ Gender (males) } & $n=35$ & $n=43$ & & \multirow[t]{2}{*}{$0.497^{\beta}$} \\
\hline & $21.0(60 \%)$ & $22(51 \%)$ & $0.09(-0.26,0.54)$ & \\
\hline \multirow{2}{*}{ Gestation (weeks) } & $n=35$ & $n=43$ & $-1.44(-1.93,-0.93)$ & \multirow{2}{*}{$<0.001$} \\
\hline & $24.54(1.27)$ & $25.98(0.98)$ & & \\
\hline \multirow{2}{*}{ Maternal steroids } & $n=35$ & $n=43$ & $0.08(-0.68,2.65)$ & \multirow{2}{*}{$0.210^{\beta}$} \\
\hline & $33.0(94 \%)$ & $37.0(86 \%)$ & & \\
\hline \multirow{2}{*}{ Maternal age } & $n=35$ & $n=43$ & $-6.19(-16.62,4.24)$ & \multirow[t]{2}{*}{0.241} \\
\hline & $27.97(4.42)$ & $30.16(30.47)$ & & \\
\hline \multirow{2}{*}{ Lowest mean arterial pressure } & $n=35$ & $n=43$ & $-4.77(-7.01,-2.49)$ & \multirow{2}{*}{$<0.001$} \\
\hline & $22.0(4.92)$ & $26.75(3.87)$ & & \\
\hline \multirow{2}{*}{ Highest blood lactate in the first 12 hours } & $n=30$ & $n=42$ & $0.34(-1.11,1.80)$ & \multirow{2}{*}{0.121} \\
\hline & $6.44(3.32)$ & $6.10(2.74)$ & & \\
\hline \multirow{2}{*}{ CRIB score } & $n=35$ & $n=43$ & $3.51(1.92,5.10)$ & \multirow[t]{2}{*}{$<0.001$} \\
\hline & $7.25(4.03)$ & $3.74(2.67)$ & & \\
\hline \multirow{2}{*}{ FT4 (pmol/L) } & $n=34$ & $n=40$ & $-7.19(-9.81,-4.58)$ & \multirow{2}{*}{$<0.001$} \\
\hline & $6.48(4.01)$ & $13.61(5.01)$ & & \\
\hline \multirow{3}{*}{ TSH (mU/L) } & $n=34$ & $n=40$ & $-0.78(-1.92,0.36)$ & \multirow{3}{*}{$0.177^{\#}$} \\
\hline & Median 2.21 & Median 2.98 & & \\
\hline & $\operatorname{IQR}(1.1,3.4)$ & $\operatorname{IQR}(2.32)$ & & \\
\hline \multirow{2}{*}{ TT4 (nmol/L) } & $n=30$ & $n=28$ & $-33.68(-51.49,-15.86)$ & \multirow{2}{*}{0.018} \\
\hline & $46.22(33.60)$ & $79.90(32.87)$ & & \\
\hline \multirow{2}{*}{ TT3 (nmol/L) } & $n=17$ & $n=20$ & $-0.38(-0.58,-0.18)$ & \multirow{2}{*}{0.012} \\
\hline & $0.40(0.22)$ & $0.77(0.35)$ & & \\
\hline \multirow[t]{2}{*}{ Cortisol (nmol/L) } & $n=34$ & $n=40$ & 219.26 & \multirow[t]{2}{*}{0.380} \\
\hline & $521.67(454.80)$ & $480.40(192.50)$ & $(54.44,384.08)$ & \\
\hline
\end{tabular}

Data expressed as mean (SD) for continuous parametric outcomes, Student's $t$-test; $x$ (\%) for categorical outcomes, ${ }^{\beta}$ chi-square, median, and interquartile ranges (IQR) for nonparametric outcomes; ${ }^{\#}$ Mann-Whitney $U$ and difference in medians calculated using Hodges-Lehmann estimate and Moses distribution free $95 \%$ confidence interval.

TABLE 2: Multivariable regression analyses of factors affecting FT4: lowest MAP, dopamine exposure, CRIB score, gestation, and highest plasma lactate concentration.

\begin{tabular}{lccc}
\hline $\begin{array}{l}\text { Predictor } \\
\text { variables }\end{array}$ & $B$ coefficient & $\begin{array}{c}\text { 95\% confidence } \\
\text { interval for } B\end{array}$ & $P$ value \\
\hline $\begin{array}{l}\text { Lowest MAP } \\
\text { (mm Hg) }\end{array}$ & 0.13 & -0.21 to 0.46 & 0.46 \\
$\begin{array}{l}\text { Dopamine } \\
\text { CRIB score }\end{array}$ & -3.77 & -7.29 to -0.25 & 0.03 \\
$\begin{array}{l}\text { Gestation } \\
\text { (weeks) }\end{array}$ & -0.31 & 0.04 to 1.09 & 0.04 \\
$\begin{array}{l}\text { Highest lactate } \\
\text { (mmol/L) }\end{array}$ & -0.23 & 1.22 to 4.45 & 0.01 \\
\hline
\end{tabular}

Stepwise model: dependent variable FT4, predictor variables entered: lowest MAP, dopamine, CRIB score, gestation, and highest lactate. Constant intercept $=17.26$. Adjusted $R$ square $=0.72 ; F=72.6, P=0.01$ (using stepwise model).

levels were not significantly different between the two groups, supporting the hypothesis that dopamine does not act only at the level of the hypothalamus and pituitary. Thus, the developing human appears to show differences compared to animal models, where Cooper et al. [6] showed a direct effect of dopamine on thyrotroph cultured bovine pituitary cells, but, unlike Foord et al. [15], no effect of dopamine on basal TSH levels but only on TRH-mediated TSH secretion [6].

One of the limitations of this study was that values of lactate concentrations and MAP were not sequential and only reported as extremes as it was not possible to assess accurately the durations of the variations in lactate and MBP. However, the extremes of MBP and plasma lactate concentrations do provide a measure of the severity of hypotension and tissue hypoperfusion. All babies were receiving intensive care at the time of the study. The condition of an infant when stressed may affect the cortisol hormone concentrations but all babies were clinically stable at the time that the blood samples were taken. However, while this small observational study adds to the current body of evidence relating to a possible interaction between the administration of dopamine and the secretion of thyroid hormone, what is needed is a randomised controlled trial of dopamine and dobutamine that considers the overall 
benefit risk ratio of each agent taking account of endocrine factors.

The mechanisms for the effect of dopamine exposure on plasma FT4 concentrations are unclear but this study has shown that it did not appear to be related to hypotension or tissue underperfusion using plasma lactate levels as a marker of organ hypoxia. The lack of an association between dopamine treatment and serum cortisol levels is also an important and clinically relevant finding suggesting that the hypothalamo-pituitary-adrenal axis was unaffected. In line with previous studies, the results of this study suggest that the effect of dopamine appears to be directly on the thyroid gland and unrelated to the indications for dopamine administration, namely, hypotension or tissue hypoperfusion. Thus dopamine administration may further directly aggravate the well described phenomena of hypothyroxinaemia of prematurity described in extremely premature infants [16]. Although the longer term consequences of such disruption remain to be elucidated and the mechanisms are unclear, these findings need to be kept in mind when administration of the vasopressor-inotrope dopamine is being considered for the treatment of neonatal cardiovascular compromise. Importantly, when administration of an inotrope is warranted, dobutamine has been shown to be devoid of significant effects on the pituitary and thyroid glands [3]. The results suggest that the effect of dopamine is directly on the thyroid gland and not related to hypotension or tissue underperfusion.

\section{Ethical Approval}

Ethical approval was obtained from UK North West Research Ethics Committee 07/MRE08/37, approval 20/092007.

\section{Conflict of Interests}

The authors declare that there is no conflict of interests regarding the publication of this paper.

\section{Authors' Contribution}

Sze M. Ng, Mark A. Turner, Paul Newland, and A. Michael Weindling conceived the study and participated in its methodology. Gabriella Watson assisted in the data collection and methodology. Sze M. Ng, Gabriella Watson, and A. Michael Weindling drafted the paper. All authors read and approved the final paper.

\section{Acknowledgment}

The authors would like to acknowledge UK Medical Research Council for the award of Clinical Research Fellowship grant to Dr. Sze M. Ng.

\section{References}

[1] S. M. Ng, "Hypothyroxinemia of prematurity: cause, diagnosis and management," Expert Review of Endocrinology and Metabolism, vol. 3, no. 4, pp. 453-462, 2008.
[2] S. M. Ng, M. A. Turner, C. Gamble et al., "An explanatory randomised placebo controlled trial of levothyroxine supplementation for babies born $<28$ weeks'gestation: results of the TIPIT trial," Trials, vol. 14, no. 1, article 211, 2013.

[3] L. Filippi, M. Pezzati, C. Poggi, S. Rossi, A. Cecchi, and C. Santoro, "Dopamine versus dobutamine in very low birthweight infants: endocrine effects," Archives of Disease in Childhood: Fetal and Neonatal Edition, vol. 92, no. 5, pp. F367-F371, 2007.

[4] I. Seri, T. tulassay, J. Kiszel et al., "Effect of low-dose dopamine infusion on prolactin and thyrotropin secretion in preterm infants with hyaline membrane disease," Biology of the Neonate, vol. 47, no. 6, pp. 317-322, 1985.

[5] C. Dieguez, S. M. Foord, G. R. Newman et al., "Rat anterior pituitary cells maintained on artificial capillaries: responses of thyrotrophs and lactotrophs to depolarization, TRH and dopamine," Molecular and Cellular Endocrinology, vol. 37, no. 1, pp. 73-82, 1984.

[6] D. S. Cooper, A. Klibanski, and E. C. Ridgway, "Dopaminergic modulation of TSH and its subunits: in vivo and in vitro studies," Clinical Endocrinology, vol. 18, no. 3, pp. 265-275, 1983.

[7] M. L. Reuss, N. Paneth, J. A. Pinto-Martin, J. M. Lorenz, and M. Susser, "The relation of transient hypothyroxinemia in preterm infants to neurologic development at two years of age," The New England Journal of Medicine, vol. 334, no. 13, pp. 821-827, 1996.

[8] A. G. van Wassenaer, J. Westera, B. A. Houtzager, and J. H. Kok, "Ten-year follow-up of children born at $<30$ weeks'gestational age supplemented with thyroxine in the neonatal period in a randomized, controlled trial," Pediatrics, vol.116, no. 5, pp. e613e618, 2005.

[9] G. van den Berghe, F. de Zegher, and P. Lauwers, "Dopamine suppresses pituitary function in infants and children," Critical Care Medicine, vol. 22, no. 11, pp. 1747-1753, 1994.

[10] L. Filippi, M. Pezzati, A. Cecchi, and C. Poggi, "Dopamine infusion: a possible cause of undiagnosed congenital hypothyroidism in preterm infants," Pediatric Critical Care Medicine, vol. 7, no. 3, pp. 249-251, 2006.

[11] F. de Zegher, G. van den Berghe, H. Devlieger, E. Eggermont, and J. D. Veldhuis, "Dopamine inhibits growth hormone and prolactin secretion in the human newborn," Pediatric Research, vol. 34, no. 5, pp. 642-645, 1993.

[12] G. Parry, J. Tucker, and W. Tarnow-Mordi, "CRIB II: an update of the clinical risk index for babies score," The Lancet, vol. 361, no. 9371, pp. 1789-1791, 2003.

[13] F. L. R. Williams, S. A. Ogston, H. Van Toor, T. J. Visser, and R. Hume, "Serum thyroid hormones in preterm infants: associations with postnatal illnesses and drug usage," Journal of Clinical Endocrinology and Metabolism, vol. 90, no. 11, pp. 59545963, 2005.

[14] S. M. Ng, D. Anand, and A. M. Weindling, "High versus low dose of initial thyroid hormone replacement for congenital hypothyroidism," Cochrane Database of Systematic Reviews, no. 1, 2009.

[15] S. M. Foord, J. R. Peters, C. Dieguez, M. F. Scanlon, and R. Hall, "Dopamine receptors on intact anterior pituitary cells in culture: functional association with the inhibition of prolactin and thyrotropin," Endocrinology, vol. 112, no. 5, pp. 1567-1577, 1983.

[16] F. L. R. Williams, T. J. Visser, and R. Hume, “Transient hypothyroxinaemia in preterm infants," Early Human Development, vol. 82, no. 12, pp. 797-802, 2006. 


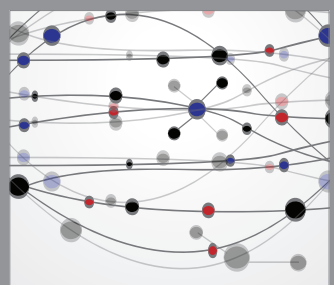

The Scientific World Journal
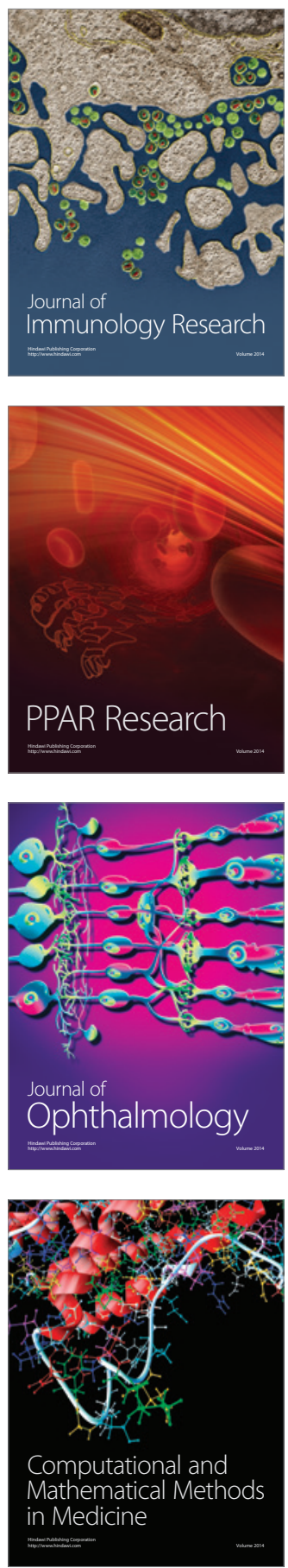

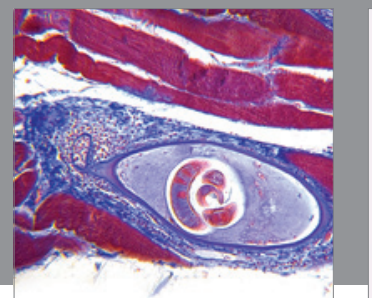

Gastroenterology

Research and Practice
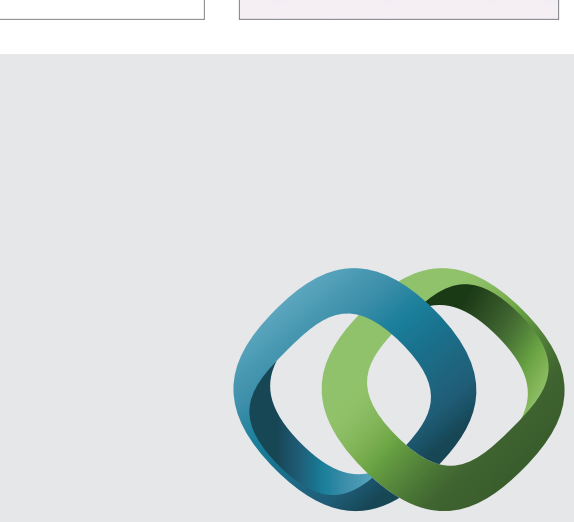

\section{Hindawi}

Submit your manuscripts at

http://www.hindawi.com
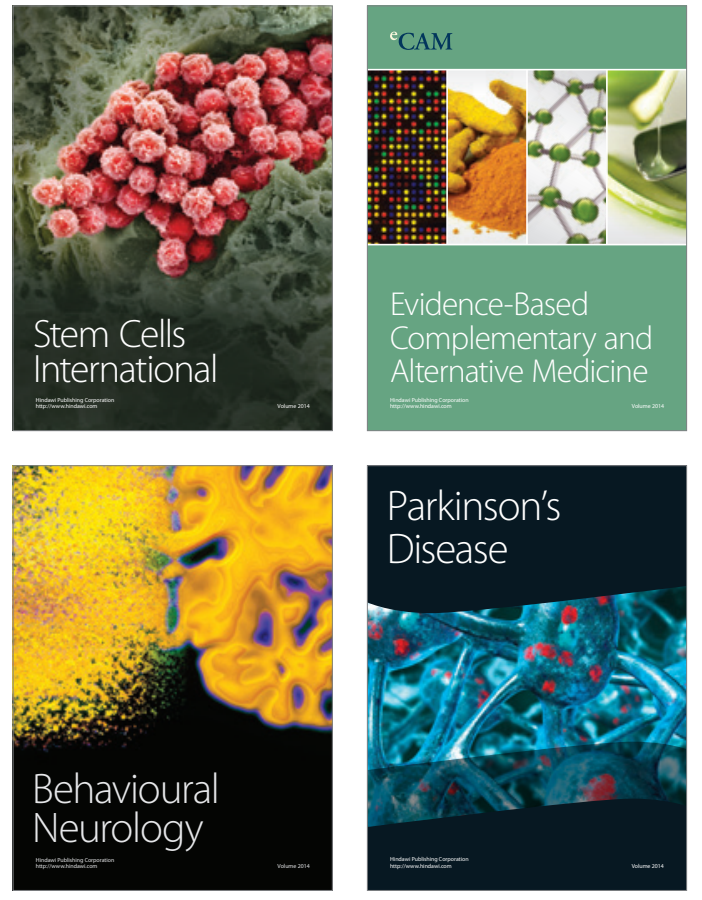
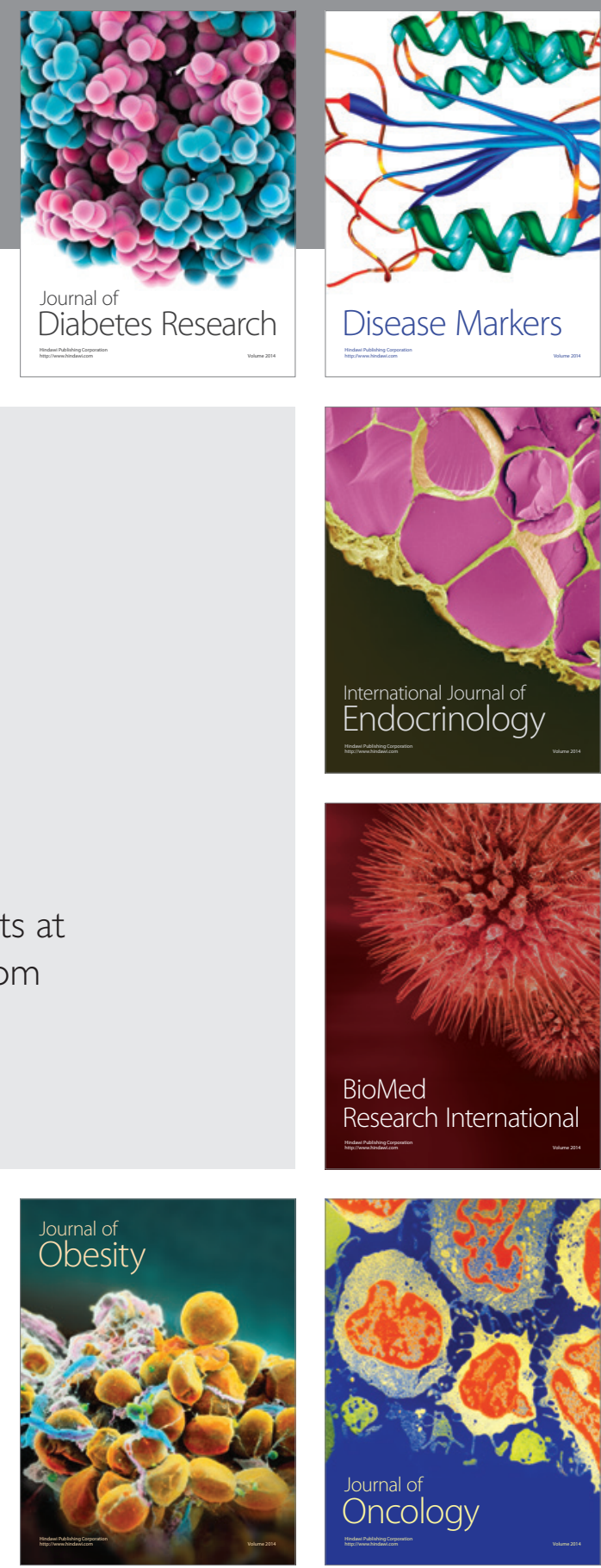

Disease Markers
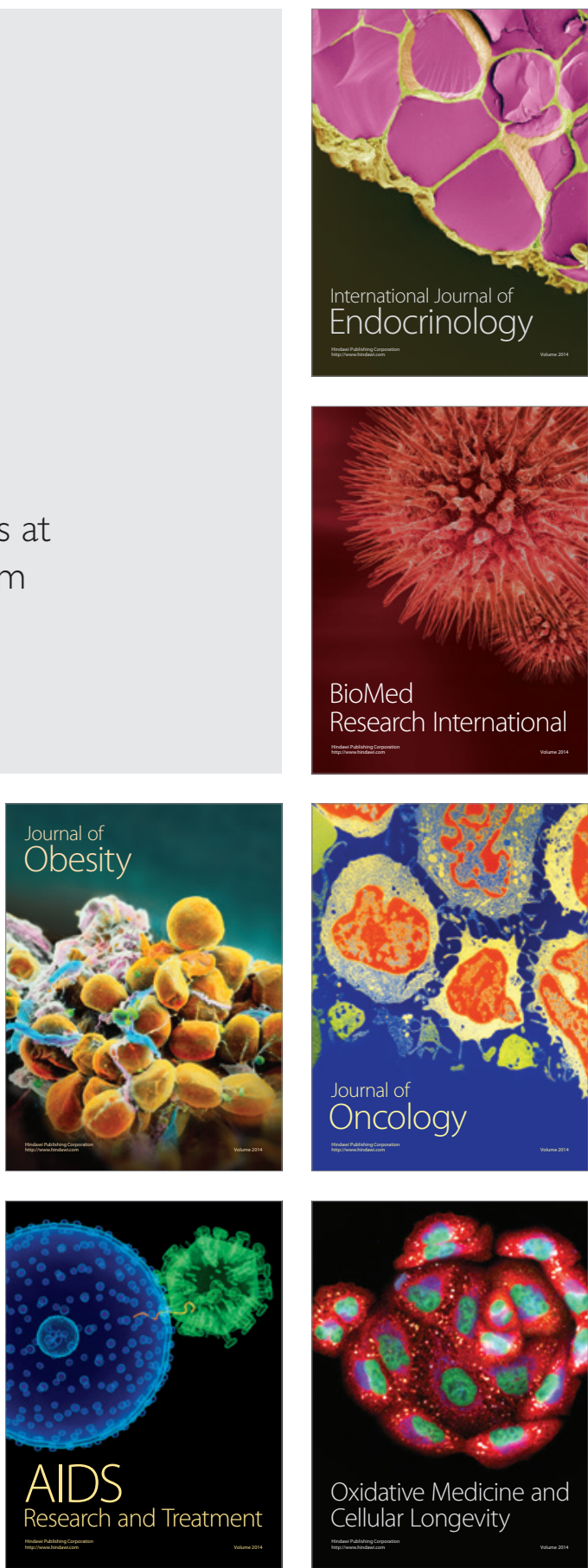